\title{
Teoría Jurídico - Económica del Daño Moral para la Indemnización de la Responsabilidad Estatal ${ }^{53}$
}

\author{
Legal - Economic Theory of Moral Damage for the Compensation of State Responsibility \\ ${ }^{a}$ Valentina Manotas López, Elfa Luz Mejía Mercado ${ }^{b}$ \\ avalentina_manotas@hotmail.com “Semillero Políticas Públicas Participación y Desarrollo, Programa de Derecho, Universidad de Cartagena. \\ Cartagena, Colombia. \\ balentina_manotas@hotmail.com “Grupo de Investigación Conflicto y Sociedad, Programa de Derecho, Universidad de Cartagena. Cartagena, \\ Colombia.
}

Forma de Citar: V.M. López “Teoría Jurídico - Económica del Daño Moral para la Indemnización de la Responsabilidad Estatal”, Rev. Saberes, Vol. 13, No. 02, pp. $68-74,2020$.

Recibido: 24/03/2020 Evaluación: 28/05/2020 Aceptado: 30/06/2020 DOI: https://doi.org/1025213/1794-4384/1302.0009

\section{Resumen}

La imprecisión de las altas cortes en Colombia al momento de la cuantificación del daño moral debido a la falta de unificación de estos a la hora de ser indemnizados por su complejo criterio de valoración, genera en muchos casos un doble pago de ellos en materia de responsabilidad estatal y evidencia un derroche de dinero que contribuye al aumento del déficit monetario que el Estado colombiano posee debido a la exorbitante deuda pública. Contrariando así teorías monetarias como las de la escuela francesa post keynesiana la cual estima los gastos presupuestales del Estado estabilizadores automáticos capaces de oponerse al riesgo sistemático. A partir de ello podría afirmarse que el Estado contribuye, mediante la no unificación de los daños morales o la incorrecta globalización de ellos, a la responsabilidad aquiliana creando un riesgo sistemático económico, lo que crea la necesidad de una teoría jurídico-económica que propenda por una relación costo-beneficio mediante la correcta globalización de los daños morales. ¿Cómo evitar el desequilibrio económico a la hora de indemnizar daños morales en materia de responsabilidad estatal propendiendo por una relación costobeneficio mediante una correcta unificación de estos daños?

\section{Palabras Clave}

Daño, economía, indemnización, estatal.

\begin{abstract}
The imprecision of the high courts in Colombia at the moment of the quantification of the nonmaterial damage (moral damage) to the lack of unification of these at the moment of being indemnified by its complex criterion of valuation generates in many cases a double payment of them as for state responsibility and demonstrates a waste of money that contributes to the increase of the monetary deficit that the Colombian possesses due to the exorbitant national debt. Contradicting monetary theories such as of the post Keynesian French school which estimates the budget expenses of the state automatics capable of being opposed to the systematic risk, from it we could say that the state contributes, by the nonunification of the moral damage or the incorrect globalization of them, to the torts of the state that creates a systematic economic risk. Its necesary a juridical - economic theory that tends for a relation cost-benefit that means a correct globalization of the moral damage. How to avoid the economic imbalance at the moment of indemnify nonmaterial damage as for state liability looking for a
\end{abstract}

\footnotetext{
* Autor para correspondencia: correo electrónico valentina_manotas@hotmail.com

(C) 2020 Fundación Universitaria Antonio de Arévalo - UNITECNAR. Este es un artículo bajo la licencia CC BY-NC-ND (http://creativecommons.org/licenses/by nc-nd/4.0/).

${ }^{53}$ Ponencia presentada en el marco del I Congreso de Investigación Científica en Ciencias Jurídicas y Económicas
} 
good relation cost-benefit by a correct unification of these damages?

\section{Keywords}

Hurt, economy, indemnification, state.

\section{Precisión del Concepto de Perjuicio Moral}

Para entrar en materia es necesario clarificar lo que se entiende como perjuicio considerando pertinente la definición doctrinal de daño como "nocimiento o perjuicio, es decir, aminoración o alteración de una situación favorable" (De Cupis, 1965, p. 81). Podríamos consecuentemente afirmar que "daño o perjuicio en sentido jurídico es lo mismo" (Tamayo, 2009). Concepto que no se refiere en un sentido material o físico sino un concepto relativo a la disminución de ventaja económica o extra patrimonial no prohibida por el Estado, con la anterior precisión es necesario responder la pregunta ¿Qué abarca el perjuicio moral? Dentro del anterior podemos incluir todos "los atentados contra la vida e integridad personal, bienes jurídicos extra patrimoniales" (Henao,1999,p.249), es menester anotar que no todo perjuicio moral debe ser indemnizable sino solo cuando se lesionan las facultades jurídicas para exigir o recibir el beneficio que ha sido suprimido anotando a lo anterior que por su falta de naturaleza económica la indemnización de este tipo de perjuicio es difícil de tasar en un valor determinado para suplir las carencias que afectan la esfera más íntima de la persona, como lo son sus sentimientos, su angustia o incluso su depresión.

Es menester mencionar la aclaración que realiza el Dr. Juan Carlos Henao en su libro Responsabilidad Extracontractual del Estado donde advierte que es importante diferenciar la naturaleza de la lesión ya que cuando se lesionan derechos pecuniarios la reparación de estos es de naturaleza puramente económica contrario sensu cuando se lesionan derechos no pecuniarios que deben ser reparados en formas diversas a la económica ya que estos son prácticamente indeterminables en dinero resaltando que cuando se otorgan sumas de dinero por estos la misma no equivale al daño si no a su compensación, siendo esta reparación de mayor complejidad ya que el juez posee un "arbitrium iudicis" para la forma de reparación.

\section{1 ¿Qué considera el Consejo de estado como} perjuicio moral?

Con la finalidad de determinar los estándares de valoración que utiliza el Consejo de Estado para la tasación de perjuicios morales es pertinente analizar lo que considera como perjuicio moral esta honorable Corte, la cual en una de sus sentencias del 2016 los define como:

Los generados en el plano psíquico interno del individuo, reflejado en los dolores o padecimientos sufridos a consecuencia de la lesión a un bien y a los cuales la jurisprudencia les exige 6 elementos para ser indemnizados (i) esa indemnización se hace a título de compensación y no de restitución, ni de reparación; (ii) debe darse aplicación al principio de equidad, previsto en el artículo 16 de la Ley 446 de 1998; (iii) su cuantificación debe estar sustentada en los medios probatorios que obran en el proceso respecto del perjuicio y su intensidad, y (iv) debe estar fundamentada, cuando sea el caso, en otras providencias para efectos de garantizar el principio de igualdad. (Consejo de Estado, 2016, Ramiro Pazos Guerrero)

En lo pertinente a los daños morales consideró el Consejo de Estado en sentencia 41001233300020120020601 (15982016) del 5 de octubre 2017 que esos son dolores o padecimientos que pueden presentarse solamente como secuela de los daños infligidos a la persona, por lo que no lo consideran en si como un daño si no como un sacrificio de intereses morales que por su condición merecen un resarcimiento satisfactorio, en lo referente a la tasación de este tipo de perjuicios en esta última sentencia la corte señala que estos se reconocen a quien sufre el daño y que de resarcirse a título de indemnización más no de reparación y la tasación le corresponde establecerla al juez de manera proporcional al daño acontecido, en lo que podemos analizar que en un corto tiempo la Corte ha cambiado de criterios con respecto a la tasación de este tipo de perjuicios a la hora de determinar la responsabilidad estatal lo que posiblemente sea uno de los factores determinantes para que estas tasaciones no se realicen de la mejor forma.

Lo anterior contribuye al despilfarro del corto presupuesto estatal que maneja la nación en la actualidad debido a la deuda externa que oscila desde el año 2003 hasta el 2018 según análisis 
económicos entre el $30 \%$ y el 40,2\%, del PIB y que actualmente corresponde en cifras exactas al $39,5 \%$ lo que compromete gran parte de los ingresos nacionales y deja en claro que no estamos en condición de realizar gastos de más en ningún ámbito con la finalidad de que se mantenga un equilibrio económico estatal como el que se plantea en algunas teorías como las de la escuela francesa pos keynesiana la cual estima los gastos presupuestales del estado estabilizadores automáticos capaces de oponerse al riesgo sistemático (P1iégay, P. \& Rochon, L.2005).

En 2017 esta situación fue llamada la "Vena abierta del Estado" por la revista dinero, así catalogaron las demandas por indemnizaciones estatales encargándose de darnos una idea de sus dimensiones, "las cifras de la Agencia Nacional de Defensa Jurídica del Estado señalan que al 30 de junio de 2017 sumaban 480.893 demandas por un valor de \$332,7 billones"(La vena abierta del estado, 2017), situación que llevo a el director de la agencia en ese año Luis Guillermo Vélez a afirmar que no existían límites a estos montos y, según él, detrás de esta situación hay una "industria sofisticada" que pone en riesgo la sostenibilidad fiscal del país, es menester resaltar que en este mismo año surgen unas reflexiones doctrinales sobre el tema en el libro Nuevas Reflexiones sobre el Daño. "Lo cierto es que nuestra jurisprudencia cada día es más generosa con las víctimas no solo en cuanto a las personas que tienen derecho a la indemnización de daños morales, sino en cuanto a las sumas asignadas a cada uno de los demandantes (...) las arcas del estado cada día se ven más afectadas". (Tamayo, 2017, p. 26).

\section{2. ¿Cómo es la Valoración para la Indemnización del Perjuicio Moral por Parte del Estado?}

\subsection{Valoración del Consejo de Estado}

La jurisprudencia del Consejo de estado muestra hacia la valoración de los perjuicios morales una extrema generosidad, lo que en muchos de los casos no corresponde a los verdaderos daños probados inclusive otorga repetidamente una indemnización. Lo anterior es de notable apreciación en una de las sentencias de la subsección tercera del Consejo de Estado en febrero de 2016, en la cual se resuelve una demanda de reparación directa contra el Municipio de Itagüí, interpuesta por los padres del menor directamente afectado, con la finalidad de que se le declare administrativamente responsable de los perjuicios causados con ocasión de la pérdida del ojo derecho del menor, a pesar de ser fallada de forma positiva con respecto a la responsabilidad del estado en el caso concreto, se condena en este a indemnizar por perjuicios morales por la suma de 100 s.m.l.v. a los padres del menor y en otro punto se resuelve indemnizar por la misma razón a los hermanos del menor quienes son representados por los padres por la suma de 50 s.m.l.v. esta es una clara evidencia de que la jurisprudencia del consejo de estado muestra hacia la valoración de los perjuicios morales una extrema generosidad, lo que en muchos de los casos no corresponde a los verdaderos daños probados inclusive otorgando repetidamente una indemnización. ¿se estaría en este fallo bajo la presencia de una doble indemnización de los perjuicios morales? ¿no podrían ser estos globalizados en una única suma? La autora considera que la respuesta a los anteriores interrogantes puede y debe ser positiva debido a que la afectación tanto de los padres como la de los dos hermanos del menor se va a ver reflejada a fin de cuentas en el mismo núcleo familiar y que por consiguiente esta debería ser globalizada bajo los criterios de la proporcionalidad sin perder de vista que estos padecimientos son estados del espíritu de algún modo contingentes y variables en cada caso y que cada cual experimenta a su modo con el fin de evitar un desgaste innecesario de presupuesto estatal.

A pesar de lo anterior en otro fallo de la subsección A durante el mismo año, el criterio de la Corte fue distinto encontrándose bajo el marco del conflicto armado los actores pidieron 100 s.m.l.v., para cada uno de ellos, en atención al sufrimiento y angustia que debieron soportar y a pesar de ello sin percatarse de ello la Corte utiliza un criterio de globalización de los perjuicios morales decidiendo condenar al Estado a pagar, por perjuicios morales, 20 salarios mínimos legales mensuales para cada una de los miembros del grupo familiar demandante.

Lo anterior claramente demuestra una falta de armonización de criterios a la hora de indemnizar perjuicios morales al igual que en otros fallos del 
mismo año dejando al azahar el presupuesto nacional el cual es directamente afectado y es el objeto principal de este análisis podríamos también agregar que el criterio de fallar desmedidamente a favor a de los afectados no solo es un problema de las altas cortes colombianas y que incluso pudimos haber acogido como es habitual tendencias internacionales como la francesa para establecerle criterios a la indemnización de perjuicios morales. Un claro ejemplo es la tendencia francesa a favor de las víctimas que se hizo especialmente evidente con la promulgación de la ley del 5 de julio de 1985 conocida como ley Badinter, explícitamente concebida para mejorar la situación de las víctimas de accidentes de tránsito y agilizar los respectivos procedimientos de indemnización y la que se considera ha ampliado de forma excesiva los campos de la responsabilidad civil representando un gran avance en pro de la uniformidad de las indemnizaciones ya que extrema la teoría del riesgo, haciendo que la víctima no pueda verse privada de la correspondiente reparación, incluso cuando media culpa de su parte, salvo que sea exclusiva favoreciendo el acuerdo amistoso entre el asegurador y el perjudicado o sus herederos.

\subsubsection{La Reparación Integral}

El concepto de reparación integral que ha sido incorporado por el Consejo de Estado en Colombia es de gran avance para entender la indemnización de perjuicios morales puesto que nos ayuda a evitar limitar el concepto de indemnización a aquella pecuniaria por lo que la doctrina francesa y Juan Carlos Henao afirman que, para impedir esa confusión, lo mejor es utilizar el concepto de "reparación" que es una palabra mucho más omnicomprensiva. Por lo anterior del verbo reparar se debe entender que existen diversas formas de volver al Estado anterior y ello ha sido reiterado por la jurisprudencia colombiana.

En cuanto a los daños morales que es lo que nos concierne, en la sentencia C-163 de 2000, la Corte reiteró el deber de las autoridades judiciales de garantizar el derecho de las víctimas y perjudicados por un hecho punible a obtener una reparación integral que comprende la indemnización de tales daños, además de los perjuicios materiales. Afirmó entonces:

“(...) independientemente de la jurisdicción encargada de establecer el quantum de una indemnización de perjuicios, el operador jurídico deberá propender porque la reparación sea integral, es decir que cubra los daños materiales y morales causados, ya que a las autoridades judiciales les asiste el compromiso de investigar y juzgar los delitos, no sólo con el ánimo de protección de aquellos bienes jurídicamente tutelados de singular importancia para la comunidad, sino también para administrar justicia en forma que mejor proteja los intereses del perjudicado, quien es concretamente, el titular del bien jurídico afectado. En consecuencia, si en un caso particular la víctima o sus causahabientes estiman que el juez civil no incluyó en la liquidación de los perjuicios otros factores como los daños morales, obviamente pueden procurar que la indemnización sea efectivamente integral acudiendo a los medios que el ordenamiento jurídico establezca para el efecto."

Posición que reitera la Corte frente a los daños morales en la sentencia c-916 de 2002 en concordancia con lo que advierte de forma reiterada el Dr. Henao:

"En efecto, cuando se dice que la reparación ha de ser integral se está garantizando que es equivalente a la medida del daño de tal manera que se cumpla la función reparatoria de la indemnización a plenitud para que el perjudicado quede, si ello fuere posible, indemne. Probablemente este objetivo no se logre respecto de los daños morales, caso en el cual la indemnización de tales daños adquiere una función compensatoria”.

\subsection{Valoración Chilena}

De los fallos que fue posible analizar, por parte de la autora, se encontró un patrón general en las decisiones de los altos tribunales chilenos difiriendo estos a los de Colombia ya que en Chile se percibe una línea homogénea entre el año 2007 hasta los fallos más recientes debido a que las respectivas indemnizaciones por perjuicios morales se realizan de manera individual.

Un claro ejemplo del 2007 es el fallo 116 del tribunal de la Corte de Apelaciones de Concepción del ministro Carlos Álvarez Cid el 26 de septiembre de 2007 (ROL 3530 - 2004) Los hijos de la fallecida, demandaron al Fisco de Chile por el fallecimiento de está, en el Hospital Regional Guillermo Grant Benavente debido a la omisión de 
debido servicio hacia ella, que originó el fallecimiento de la madre, provocando graves dolores físicos y morales a sus familiares. Se condena al Servicio de Salud Concepción a pagar a Lisandro Gabriel Alejandro Peña Contreras a título de daño moral que personalmente sufriera, la suma de $\$ 10.000 .000$, a Luis Alberto Pérez Espinoza a título de daño moral que personalmente sufriera, la suma de $\$ 20.000 .000$, a Jessica Roxana Pérez Contreras a título de daño moral que personalmente sufriera, la suma de $\$ 20.000 .000$, y a Juan Luis Pérez Contreras a título de daño moral que personalmente sufriera, la suma $\$ 20.000 .000$ otro claro ejemplo más reciente del 28 de noviembre de 2018 demuestra que Chile ha seguido con esta misma línea sin mostrar los vestigios que son palpables en la jurisprudencia colombiana en la sentencia Rol Nº11889-2015, tramitados ante el $28^{\circ}$ Juzgado Civil de Santiago, que tramita una demanda de indemnización de perjuicios por la muerte de don Juan Carlos Cerda Sotelo, por responsabilidad contractual, la cónyuge e hijos de la víctima, en contra del Hospital Clínico de la Universidad de Chile. Este Fallo condena a la demandada a pagar indemnización por daño moral para doña Eliana Hernández Muñoz por la suma de \$25.000.000, para el actor don Juan Ignacio Cerda Hernández por $\$ 10.000 .000$ y para doña Jacqueline Cerda Hernández por $\$ 30.000 .000$. que de igual manera podemos notar que infringe la teoría planteada indemnizando como se hace comúnmente de manera individual a pesar de ser el mismo núcleo familiar lo que implica que el estado está derrochando dinero estatal indemnizando repetidamente dolores comunes que por pertenecer a un mismo núcleo familiar podrían ser los mismos, sería posible mermar el despilfarro estableciendo una suma general en la cual se tenga en cuenta las aflicciones de cada uno de los integrantes del núcleo familiar de manera individual y las que como núcleo familiar puedan compartir haciendo evidente que Chile a lo largo de los años ha demostrado que posee un desarrollo jurisprudencial en general mucho más de avanzada que otros países latinoamericanos incluyendo Colombia; sin embargo con este supuesto podríamos afirmar que a pesar de no tener una decisión unánime con respecto al tema planteado en la jurisprudencia colombiana claramente si se pueden percibir algunos vestigios de las ideas planteadas en esta tesis debido a que en algunas sentencias se encuentra aplicado el criterio de globalización para los daños morales en los mismos núcleos familiares afectados evitando ser indemnizados de forma repetida.

\subsection{Valoración Española}

En la jurisprudencia española se contempla como un referente para la indemnización del daño extra patrimonial la sentencia del 7 de febrero de 1962 en la cual el Tribunal Supremo español esboza el siguiente criterio:

"El dinero no puede aquí cumplir su función de equivalencia como en materia de reparación de daño material, la víctima del perjuicio moral padece dolores, y la reparación sirve para establecer el equilibrio roto, pudiendo gracias al dinero, según sus gustos y temperamento, procurarse sensaciones agradables, o más bien revistiendo la reparación acordada al lesionado, la forma de una reparación satisfactoria puesta a cargo del responsable del perjuicio moral, en vez del equivalente del sufrimiento moral".

La doctrina española además es unánime en afirmar que la reparación de los daños morales encuentra su fundamente base en el principio de la reparación integral del daño surgiendo este desarrollo respecto al tema desde el sistema de responsabilidad extracontractual analizando las circunstancias pasadas de la víctima (Mayor, 2015,p.9) tal cual como se empezó a descubrir la existencia del daño extra patrimonial en Colombia con la reconocida sentencia Villaveces de la sala de casación civil del 21 de julio de 1922 en la cual el municipio de Bogotá es declarado responsable patrimonialmente de los daños morales sufridos por el señor León Villaveces, al haber extraído de manera indebida y sin su respectiva autorización los restos de su esposa enterrados en un mausoleo de su propiedad.

Cabe resaltar que, debido a una buena apropiación del concepto de reparación integral por parte de la jurisprudencia española, España ha logrado reconocer nuevos daños extra patrimoniales como lo son: «perjuicio social», «perjuicio juvenil» o el daño a la «capacidad matrimonial». Aspecto que así como la globalización para una correcta indemnización del daño extra patrimonial es de gran avance a la hora de justas indemnizaciones estatales que competan este ámbito del derecho. 
Sumado a lo anterior en el Consejo General del Poder Judicial de España encontramos un panorama muy de avanzada con respecto a las indemnizaciones de daños morales por parte del estado ya que en la jurisprudencia española se cumple con condiciones para llevar acabo la teoría antes planteada debido a que un patrón general que se percibe en esta jurisprudencia es que el daño moral se globaliza en un porcentaje incluso cuando no se cumple la condición planteada anteriormente de pertenecer al mismo núcleo familiar y además se comprueba lo mencionado con respecto al reconocimiento de nuevos daños extra patrimoniales, tal como se indemnizó moralmente por la privación colectiva de un bien a la Junta de Comunidades de Castilla-La Mancha asegurando que se debía reconocer a la parte actora el derecho a percibir una indemnización, a cargo de la Administración expropiante, por importe del 25\% del justiprecio afirmando el Tribunal Supremo (2018) "en materia expropiatoria está La Extensión del Principio de Solidaridad y la Justicia Material como Sustentos para Imponer Alimentos Pos-Ruptura de Relación Sentimental en el Ordenamiento Jurídico Colombiano mente admitido que la privación coactiva del bien ocasiona no sólo una pérdida estrictamente patrimonial, sino también un daño moral que se indemniza bajo la denominación de premio de afección."

\section{Conclusiones}

Es perceptible que la tesis planteada no se encuentra ejecutada de manera completa e idónea en ninguna de las legislaciones antes analizadas, sin embargo vemos como en Colombia el Consejo de Estado muestra vestigios, ya que no es una jurisprudencia homogénea de la globalización de los daños morales que se propone en esta tesis proyectando una solución al derroche de dinero en las indemnizaciones estatales por este tipo de daños, planteando que no se indemnice como se hace comúnmente de manera individual a pesar de ser el mismo núcleo familiar evitando que el estado despilfarre dinero indemnizando repetidamente dolores comunes que por pertenecer a un mismo núcleo familiar podrían ser los mismos.

Sería posible mermar el despilfarro estableciendo una suma general en la cual se tenga en cuenta las aflicciones de cada uno de los integrantes del núcleo familiar de manera individual y las que como núcleo puedan compartir. Sin negar la ventaja de la jurisprudencia colombiana frente a la chilena en este aspecto que no deja de ser inquietante, la jurisprudencia española con respecto al tema muestra unos rasgos más de avanzada como lo son la globalización de los daños extra patrimoniales en porcentajes incluso para colectividades que no cumplen con el requisito antes planteado en esta tesis de incidir en el mismo núcleo familiar para que los daños morales logren ser globalizados.

La anterior hipótesis se plantea propendiendo por el no aumento del déficit fiscal que es un tema de preocupación en la actualidad de nuestro país, el cual se ve afectado por un sin fin de demandas contra el estado y un incontable número de indemnizaciones que se hacen a diestra y siniestra indemnizando daños ya indemnizados y así afectando el capital público colombiano que además carga a cuestas una deuda publica anual que consume gran parte del PIB nacional creando un riesgo sistemático, dejando plasmadas únicamente en el papel teorías monetarias como las de la escuela francesa pos keynesiana que considera los gastos presupuestales del estado estabilizadores automáticos capaces de oponerse al riesgo sistemático, por ello la teoría jurídicoeconómica del daño busca propender por una relación costo-beneficio entre el afectado y el estado mediante la correcta globalización de los daños morales sin dejar al estado con más afectaciones en el presupuesto estatal de las que ya posee.

\section{Referencias Bibliográficas}

Corte constitucional sentencia C-916 de 2002, expediente D-4020.Magistrado ponente: Manuel José Cepeda Espinosa.

Corte constitucional sentencia C-163 de 2000, expediente D-2486.Magistrado ponente: Fabio Morón Díaz.

Consejo de Estado, sección tercera subsección B, sentencia 05001233100019990205901 (40057), oct.3/16. Consejero Ponente: Ramiro Pazos Guerrero. 
Consejo de Estado, Sección Segunda, Sentencia 41001233300020120020601 (15982016), oct. 5/17. Consejero ponente: Sandra Lisset Ibarra

Consejo de Estado sala de lo contencioso administrativo sección tercera, subsección a, sentencia 19001233100020020021601 (29.299), feb.24/16 consejero ponente: Carlos Alberto Zambrano Barrera.

Consejo de estado sala de lo contencioso administrativo sección tercera subsección c, sentencia 520012331000200101210 01 (29.139), nov.12/14 consejero ponente: Jaime Orlando Santofimio Gamboa.

De Cupis A. El Daño. Bosch. Barcelona. 1996. Deuda externa de Colombia (2018), Revista dinero, disponible en : https://www.dinero.com/economia/articulo/deuda -externa-de-colombia-en-enero-de-2018/257090

¿El perjuicio moral se reconoce a manera de indemnización o de reparación? (2018), Revista Ámbito jurídico, disponible en: https://www.ambitojuridico.com/noticias/adminis trativo/administrativo-y-contratacion/el-perjuiciomoral-se-reconoce-manera-de

Henao Juan Carlos. Responsabilidad extracontractual del estado. Universidad Externado de Colombia. 2015. .39.

Henao Juan Carlos. Responsabilidad extracontractual del estado. Universidad Externado de Colombia. 2015.41.

Henao Juan Carlos. Tipología del daño. Universidad Externado de Colombia. 1999.249.

Indemnizaciones por demandas, la vena abierta del Estado (2018),Revista Dinero, disponible en: https://www.dinero.com/edicionimpresa/caratula/articulo/indemnizaciones-delestado-son-su-vena-abierta/248690

Juzgado Civil de Santiago, veinte de noviembre de dos mil dieciocho. Rol No11889-2015, tramitados ante el 28o juzgado de Santiago, recuperado de http://www.pjud.cl/documents/396543/0/JJ+SOB REDOSIS+CORTE.pdf/6395d126-1dbc-4199$\underline{8 \mathrm{ad} 1-14 \mathrm{c} 32 \mathrm{f} 69 \mathrm{bff} 4}$
Koteich Khatib Milagros, La indemnización del perjuicio extra patrimonial (derivado del "daño corporal") en el ordenamiento francés (2010) Revista de Derecho Privado.

Luis Moreno Fernández, Un baremo europeo de valoración del daño corporal, recuperado de http://civil.udg.edu/cordoba/com/Moreno2.htm

P1iégay, Pierre, \&amp; Rochon, Louis-Philippe. (2005). Teorías monetarias pos keynesianas: una aproximación de la escuela francesa. Problemas del desarrollo, 36(143), 33-57.

Recopilación de fallos de responsabilidad del estado administrador: periodo 2000-2010, recuperado de http://repositorio.uchile.cl/bitstream/handle/2250/ $113165 /$ de-inda_m.pdf? sequence $=1$

Roberto Mayor Gómez, Los daños morales en la responsabilidad patrimonial sanitaria: análisis jurídico y práctico de las cuestiones más problemáticas. Castilla-La mancha. 2015.

Tribunal Supremo de Madrid Sala de lo Contencioso STS 2031/2018 Recurso de Casación Contencioso-Administrativo (L.O. 7/2015) Ponente: Wenceslao Francisco Olea Godoy.

Tamayo Jaramillo Javier. Reflexiones sobre la ontología del daño y la relación entre daño y perjuicio, 2015, Revista Ámbito jurídico.

Tamayo Jaramillo Javier. Tratado de responsabilidad civil. Legis. Colombia. 2007 tomo II. 331.

Tamayo Jaramillo Javier. Nuevas reflexiones sobre el daño. Legis.2017.

Villegas Riera Ana maría. Responsabilidad extracontractual del estado: análisis del daño fisiológico o a la vida de relación, 2003. Pontificia universidad javeriana.

Yoleida Vielma Mendoza. Una aproximación al estudio del daño moral extracontractual Profesora de Derecho Civil Universidad de los Andes (Mérida- Venezuela) Doctoranda en Derecho Universidad de Salamanca (España). 